\title{
Time-domain imaging system in the terahertz range for immovable cultural heritage materials
}

Article

Accepted Version

Giovannacci, D., Chung, H.-C., Walker, G. C., Bowen, J. W., Martos-Levif, D., Brissaud, D., Cristofol, L. and Melinge, Y. (2019) Time-domain imaging system in the terahertz range for immovable cultural heritage materials. Strain, 55 (2). e12292. ISSN 1475-1305 doi: https://doi.org/10.1111/str.12292 Available at https://centaur.reading.ac.uk/78210/

It is advisable to refer to the publisher's version if you intend to cite from the work. See Guidance on citing.

To link to this article DOI: http://dx.doi.org/10.1111/str.12292

Publisher: Wiley

All outputs in CentAUR are protected by Intellectual Property Rights law, including copyright law. Copyright and IPR is retained by the creators or other copyright holders. Terms and conditions for use of this material are defined in the End User Agreement.

www.reading.ac.uk/centaur 
Central Archive at the University of Reading

Reading's research outputs online 


\title{
Time Domain Imaging (TDI) system in the terahertz (THz) range for immovable cultural heritage materials
}

\author{
D. Giovannacci ${ }^{1,2}$, H.-C. Chung ${ }^{1,2}$, G. C. Walker ${ }^{4}$, J.W. Bowen ${ }^{4}$, D. Martos-Levif ${ }^{3}$, D. Brissaud ${ }^{1,2}$, \\ L. Cristofol ${ }^{5}, Y$. Mélinge ${ }^{5}$ \\ ${ }^{1}$ LRMH, Laboratoire de Recherche des Monuments Historiques, Ministère de la Culture et de la \\ Communication, 29 rue de Paris, 77420 Champs-sur-Marne, France \\ ${ }^{2}$ Sorbonne Universités, Centre de Recherche sur la Conservation (CRC, USR 3224), Muséum national \\ d'Histoire naturelle, Ministère de la Culture et de la Communication, CNRS; CP21, 36 rue Geoffroy- \\ Saint-Hilaire, 75005 Paris, France \\ ${ }^{3}$ C2RMF, Centre de recherche et de restauration des musées de France, 14 quai François Mitterrand, \\ 75001 Paris, France \\ ${ }^{4}$ Biomedical Engineering, School of Biological Sciences, University of Reading, Reading, England \\ ${ }^{5}$ L2MGC, Laboratoire de Mécanique et Matériaux du Génie Civil - EA 4114, Université de Cergy- \\ Pontoise - 5 Mail Gay Lussac, Neuville sur Oise - 95031 Cergy-Pontoise Cedex
}

Abstract:

In the field of Cultural Heritage science, the use of non-destructive and contact-free techniques has increased sharply over the past 10 years. Compared to conventional spectroscopic and imaging techniques such as X-ray, Ultraviolet (UV), Infrared (IR) and laser spectroscopy, terahertz time-domain imaging (THz-TDI) is an innovative, non-invasive and safe technique, which provides good penetration depth $(\sim 1 \mathrm{~cm})$ and broad spectral bandwidth $(0.1-10 \mathrm{THz})$. This paper sets out the protocol and methodology for the application of THz-TDI to immovable Cultural Heritage, illustrated by a series of case studies. The case studies demonstrate the efficacy of the technique in providing structural and material information for conservators.

Keywords: Terahertz, time domain, immovable cultural heritage, case studies 


\section{Introduction}

Terahertz $(\mathrm{THz})$ radiation, also known as sub-millimeter wave radiation, lies between the microwave and infrared bands of the electromagnetic spectrum, Figure 1 . The wavelength of $\mathrm{THz}$ waves ranges from $30 \mu \mathrm{m}$ to $3 \mathrm{~mm}$. THz radiation exhibits properties of both millimeter radio waves and infrared light waves and can provide information about both the outside and inside of a sample [1-3]:

- In similarity to microwaves: THz radiation is transmitted through a wide variety of optically opaque and non-conducting materials (wood, plastics, ceramics, paper, bones etc.). This is an attractive feature for a wide range of applications.

- In similarity to visible light waves: THz radiation can be propagated through space, reflected, focused and refracted using $\mathrm{THz}$ optics.

Almost all materials show their own particular spectral features in the $\mathrm{THz}$ range due to the motion of entire molecules, which include phonon interaction, molecular rotations, hydrogen bond behavior and various low-energy excitation modes [1]. THz waves are non-ionizing, as the photon energies are insufficient to change the structure or properties of the material. Furthermore, $\mathrm{THz}$ radiation has limited penetration (strong absorption) in water and cannot penetrate metal [1]. The short wavelength (between $30 \mu \mathrm{m}$ and $3 \mathrm{~mm}$ ) allows for a spatial resolution that is sufficient for imaging applications.

Until the beginning of the 1990's, the THz domain (the so-called "THz gap") had not been intensively studied due to the lack of available detectors and sources prior to the introduction of femto-second lasers. Since then, THz-spectroscopy has rapidly developed for various research fields as a novel, noninvasive examination method, firstly being applied in the fields of medicine [4] and security [5]. Compared to other electromagnetic waves, such as X-ray, Ultraviolet (UV) and Infrared (IR) waves, THz radiation can provide information about sub-surface regions, up to an approximate depth of one centimeter, meaning that it has the ability to penetrate thicker layers and obtain structural or layer information using reflected signals, all under safe operating conditions.

The first published study in the field of Cultural Heritage was carried out in 2006 [6], with imagery of the internal structure of an easel painting. Since then, a large number of different materials and artworks have been studied. Figure 2 illustrates the domains in the field of conservation science where $\mathrm{THz}$ imaging has been used for movable and immovable cultural heritage.

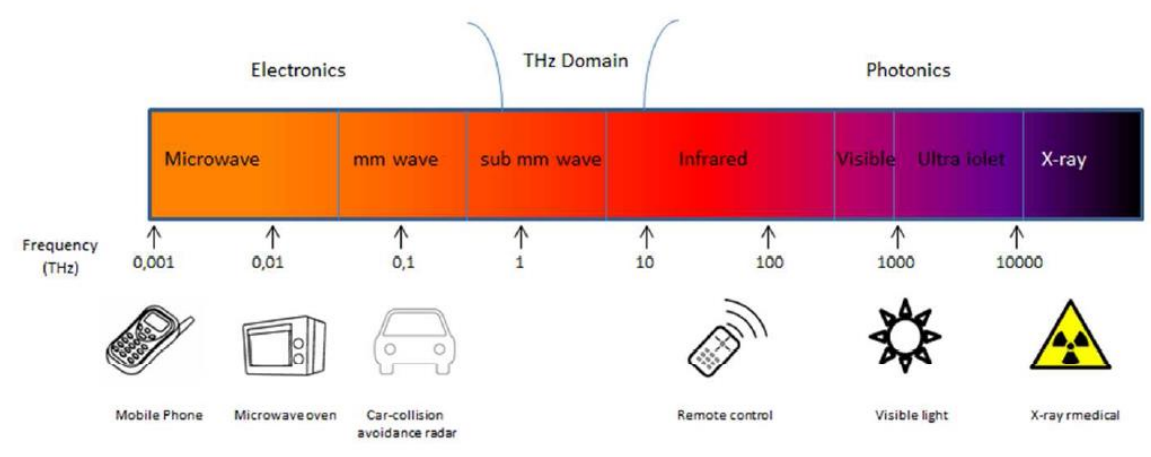

Figure 1: Electromagnetic spectral ranges in frequency and scale, indicated with the Terahertz gap. 

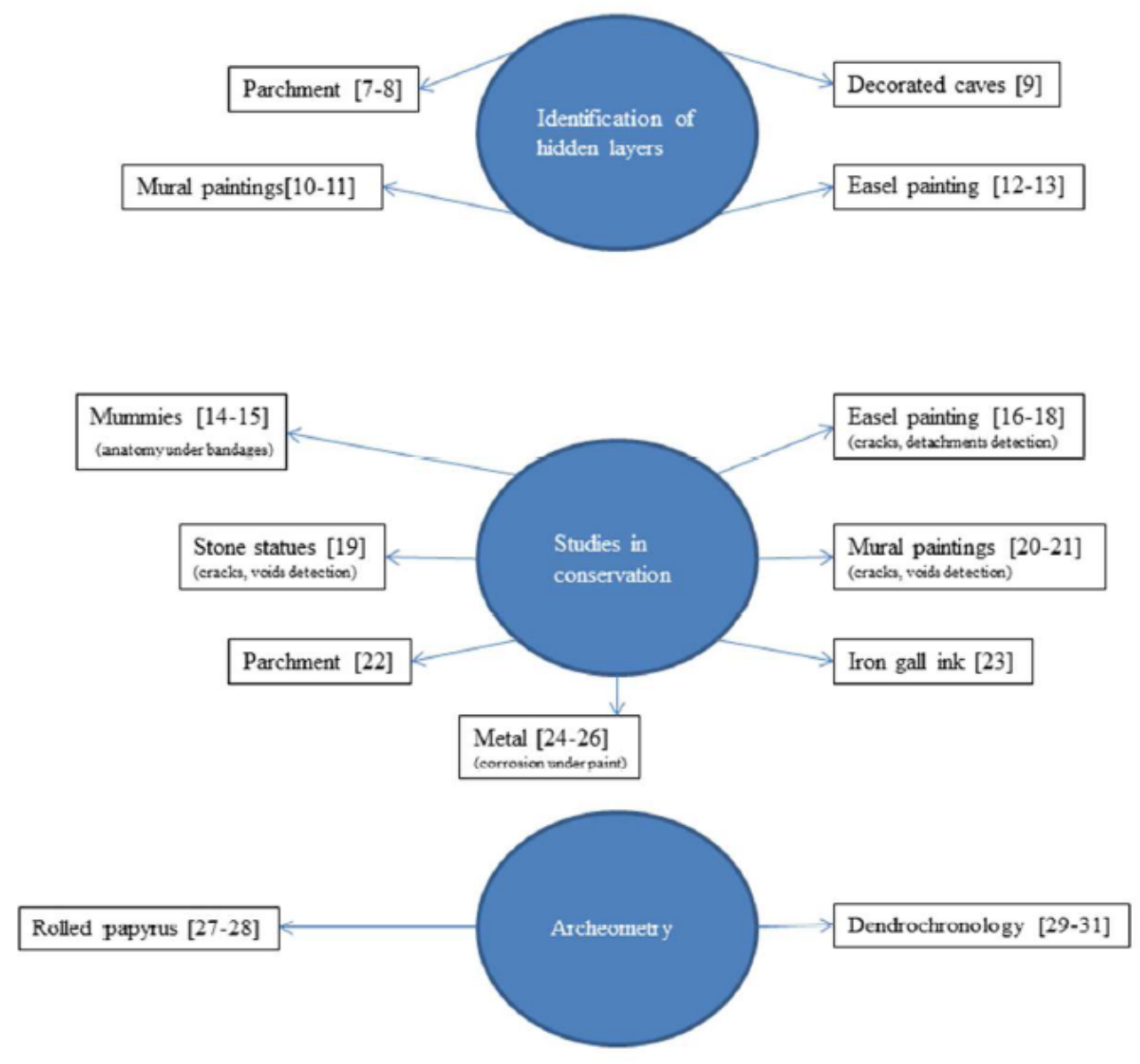

Figure 2: Applications of $\mathrm{THz}$ imaging systems in conservation science.

\section{Principles of Terahertz Time-Domain Imaging (THz-TDI)}

Terahertz Time-Domain Imaging (THz-TDI) can be used for measurement in both transmission and reflection mode, in order to identify or to highlight material structure through data processing and analysis. The imaging system allows $2 \mathrm{D}$ and $3 \mathrm{D}$ imaging (tomography and 3D reconstruction) studies with spatial resolution scaled from tens of micrometers to several millimeters [1-3].

In THz-TDI, THz-pulses are generated by applying short laser pulses to either nonlinear crystals or photoconductive antennas. Usually, a GaAs photoconductive antenna with an interdigitated electrode structure serves as a source for intense single cycle $\mathrm{THz}$ pulses when it is illuminated at normal incidence by an ultrafast laser pulse. After emission, the $\mathrm{THz}$ beam is collimated and focused on the sample with a high-density polyethylene (HDPE) lens. Then, the $\mathrm{THz}$ beam is re-collimated and the $\mathrm{THz}$ pulses detected coherently either by using electro-optical crystals or photoconductive antennas. A standard THz-TDI setup is shown in Figure 3. The detected signals are proportional to the THz electric field rather than the intensity. The system typically has a usable frequency range of approximately 0.1$3 \mathrm{THz}$ (wavelength ranging from 3 to $0.1 \mathrm{~mm}$, wavenumber ranging from 3.3 to $100 \mathrm{~cm}^{-1}$ ). The exact frequency range is linked to instrumental parameters, such as the duration and power of the femtosecond laser pulse, composition of the semiconductor material, and design of the emission and detection antennas. 


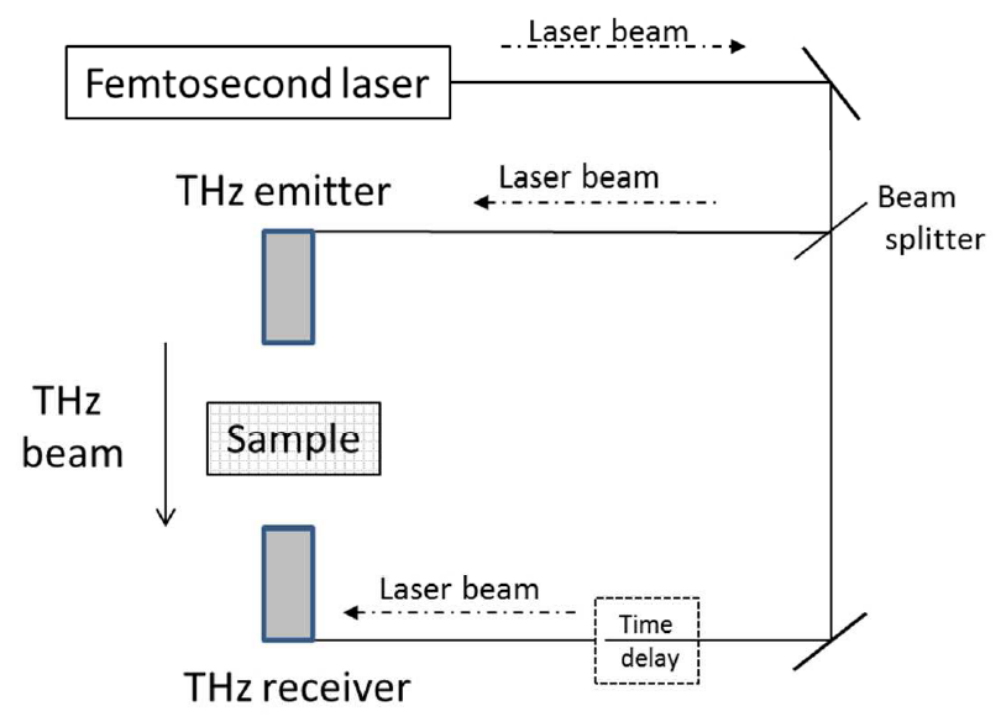

Figure 3: Schematic diagrams of simplified THz-TDI setup [1].

The data is recorded by raster-scanning the emitter/receiver configuration across the region of interest. A waveform is recorded at each position. A time-domain (TD) signal is plotted with the electrical field strength of the electromagnetic pulse as a function of time (Figure 7)). Such a signal will contain signatures corresponding to internal reflections from interfaces within a layered material, separated by their time-of-flight, and can be used to observe the distance between the layers. The positive features in the waveform correspond to interfaces where the $\mathrm{THz}$ beam has transitioned from a high-refractive-index medium to a lower refractive index medium, and vice versa for the negative features [1].

THz-TDI can be used in three specific modes: transmission, reflection with off-axis setting, and reflection with collinear setting. For the transmission mode (figure 4), the main pulse directly propagates through all the materials. The recorded data is usually transformed into the frequency domain, where it provides better signal-to-noise ratio when compared to data recorded in reflection mode. The TD signal is less used, as it cannot give temporal information on the layers.

The reflection modes provide an accurate and direct measurement of the sample thickness because the arrival time of the reflected $\mathrm{THz}$ pulse is very sensitive to any optical thickness variations in the sample. The sign and amplitude of the back-reflected $\mathrm{THz}$ pulse can be analyzed for reflections originating from the various interfaces located in the sample, with the reflection amplitude determined by the terahertz refractive index mismatch at each interface. Each pulse corresponds to structural or material property change in the sample, which results in reflection of the incident beam, even from buried layers.

In reflection mode, there are two different settings, off-axis (figure $4 \mathrm{~b}$ left) and collinear (figure $4 \mathrm{~b}$ right), which are able to highlight different characteristics and can be applied to samples with specific needs. The off-axis setting gives better signal-to-noise ratio than the collinear setting (because the recorded signal is reduced by a total of $75 \%$ due to a combination of partial transmission and partial reflection at the semi-reflective mirror inside the collinear system). However, the $\mathrm{THz}$ beam focused 
on the sample forms an elliptical spot rather than a perfect circle. In contrast, the collinear setting produces a circular spot. The off-axis system is used most often, due to the better signal-to-noise ratio obtained from the sample.

The choice of transmission and reflection mode depends on the object of the analysis and the feasibility due to the sample geometry: if it is a wall painting, transmission mode is impossible to apply, so reflection mode is the only choice. Reflection mode is also an advantage when compared to other techniques like X-ray, which can only operate in transmission.

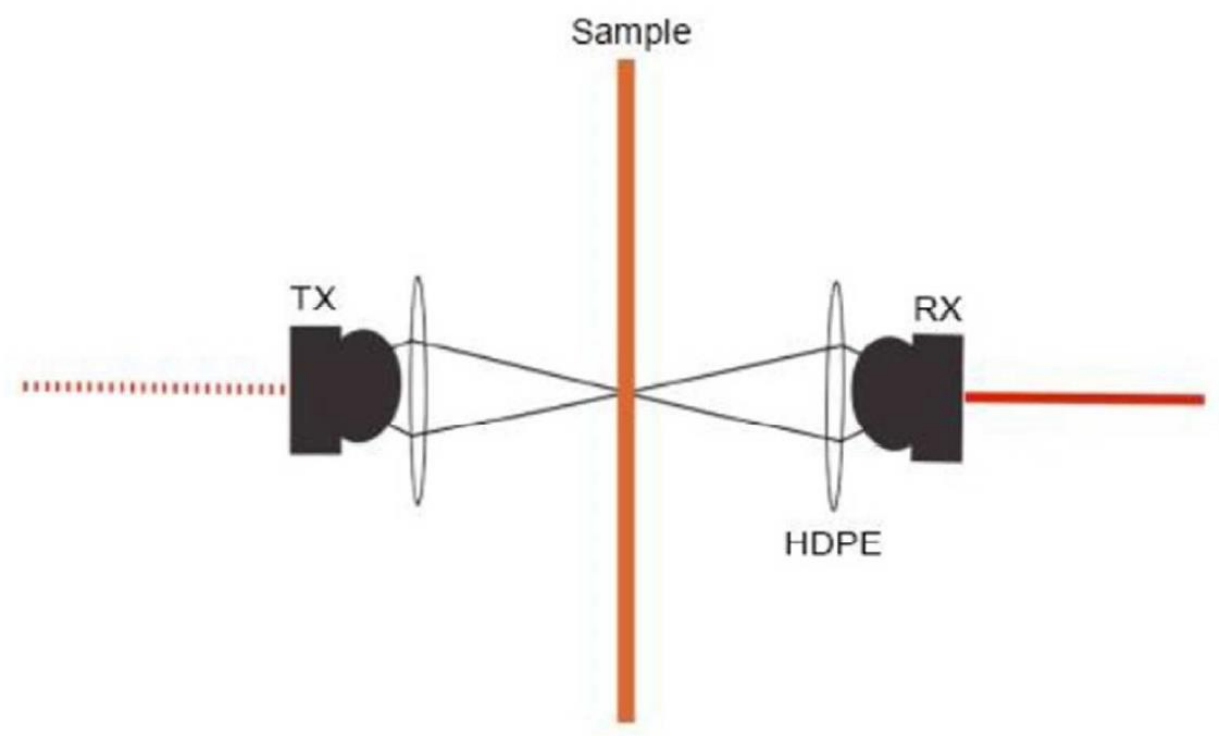

Figure 4 (a): Schematic diagram of transmission mode. TX represents the transmitter and RX the receiver.
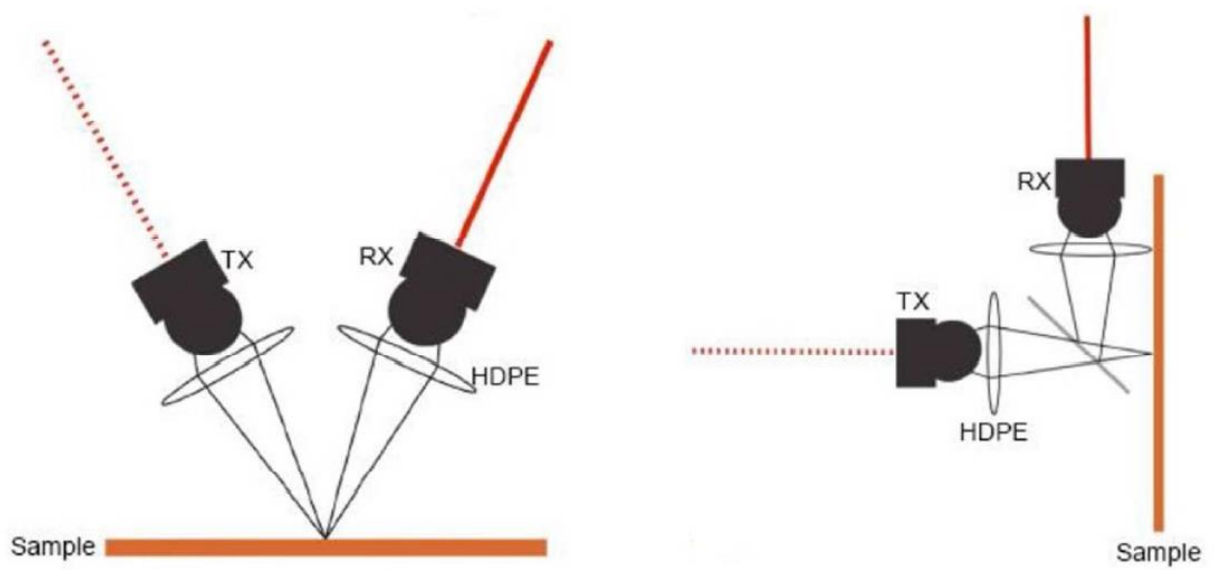

Figure 4 (b): Schematic diagrams of reflection mode: off-axis setting (left) and collinear setting (right).

\section{Materials and methods}

\subsection{Instrument and Protocol}

The experiments were conducted with a Picometrix T-Ray $4000^{\circledR}$ THz-TDS imaging system. The system consists of separate emitter and receiver heads connected by umbilical optical fibers to a box housing the ultrafast laser and associated optical components. The mode-locked, two-stage, 
amplified, Ytterbium fiber pump laser operates with a center wavelength near $1064 \mathrm{~nm}$, a $100 \mathrm{fs}$ pulse width, a $50 \mathrm{MHz}$ repetition rate and a maximum output power of $400 \mathrm{~mW}$. The emitter and receiver are low temperature GaAs photoconductive fiber-coupled antennas, allowing rapid modification of the measurement mode (transmission, off-axis and collinear reflection) for in situ analysis (Figure 5). This configuration allows a $2 \mathrm{THz}$ spectral bandwidth, with a $40 \mathrm{~dB}$ dynamic range for minimal averaging, with an acquisition rate of $100 \mathrm{~Hz}$ for a fixed $320 \mathrm{ps}$ measurement window at a $78.13 \mathrm{fs}$ time resolution. After emission, the THz beam is collimated and focused on the sample with an HDPE lens, the receiver collecting the reflected or transmitted THz pulse. All the experiments performed with reflection mode in this article used the off-axis setting.

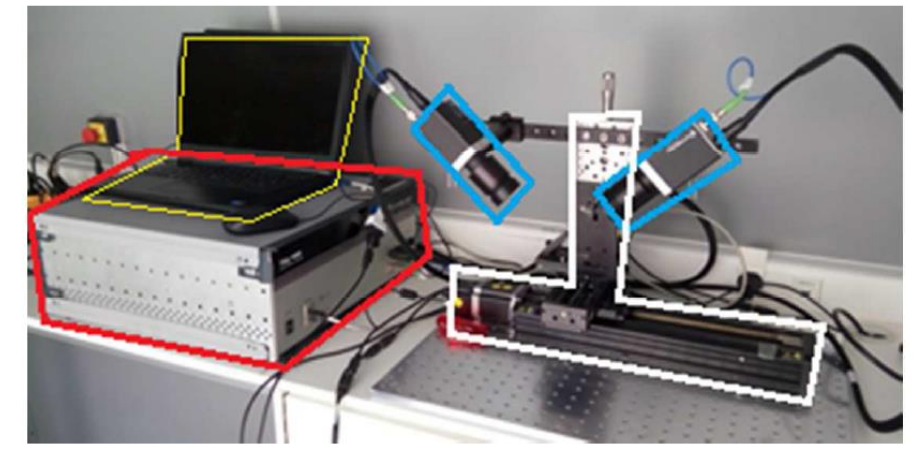

Figure 5: Picture of the THz-TDI system during in situ analysis, with computer (yellow), antennas (blue), $x-y$ translation stage (white), and laser and optics box (red).

The data is acquired by raster-scanning the emitter and receiver configuration across the region of interest (ROI). A waveform is recorded at each position, consisting of a 4096 point-time series. The parameters to optimize for the measurements include the step, the scan size and the speed, as they determine the size of each pixel and thus the resolution of the image; the scan area as defined by the region of interest; and the number of averages for each pixel, to help to improve the signal-to-noise ratio [8-9]. The acquisition time depends on the number of pixels for the ROI, the resolution of each pixel, the displacement speed and the number of averages.

\subsection{Terahertz imaging methodology}

Recording the electric field amplitude versus time for a particular position on the specimen (also called an A-scan) allows several peaks to be highlighted. Each peak reveals the difference in optical distance between the receiver and the sample surface or interfaces. This identification can provide information throughout the thickness of a specimen. A visualization of the stratigraphy can be achieved by accumulating a series of A-scans along a line (a so-called a B-scan). Thus a B-scan gives indications of stratified boundaries in the material and features inside the sample, such as cracks, air gaps or layers, with a depth resolution of better than $100 \mu \mathrm{m}[18,32]$. An illustration of such a result is given in Figures 6 and 7. The mock-up used for this experiment is shown in Figure 6 before and after having been partially covered with whitewash. The stratigraphy under the whitewash layer is depicted between the two images. In the B-scan shown in Figure 7, the horizontal axis represents the position on this line ( $Y$ coordinate) and the vertical axis represents the time increment. The color scale represents the amplitude of the signal at each position and time point in the acquisition window [13].

2D cartography, also called a C-scan, can be carried out by choosing a particular value of time of flight (corresponding to a fixed optical distance from the antennas) or a particular value of electric field amplitude, for example the maximum (which may be at a varying optical distance from the antenna). 

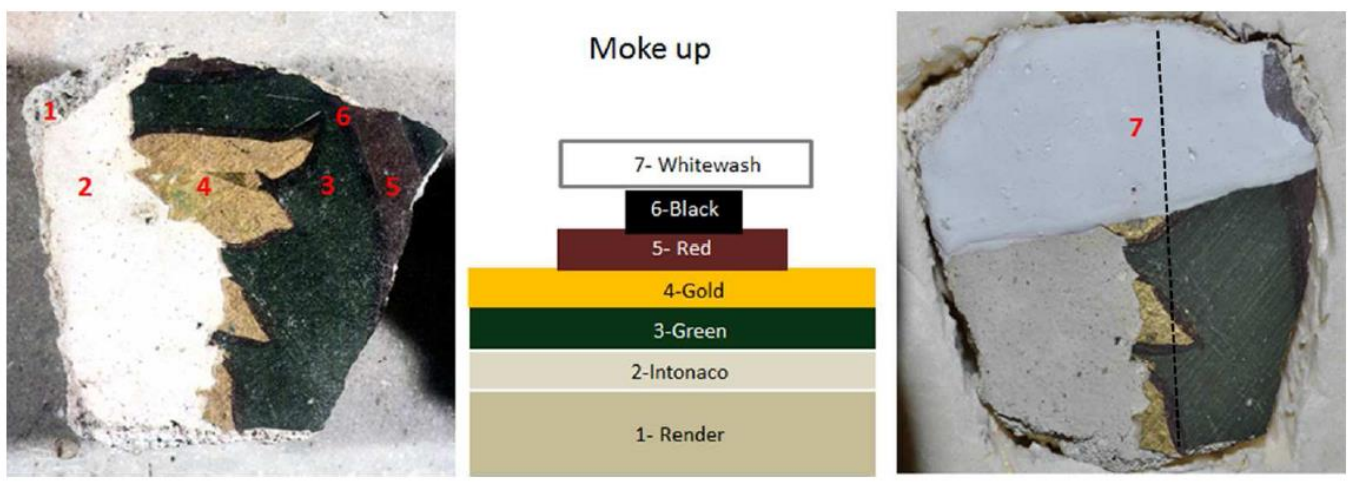

Figure 6: Sample without and with whitewash and its stratigraphy.
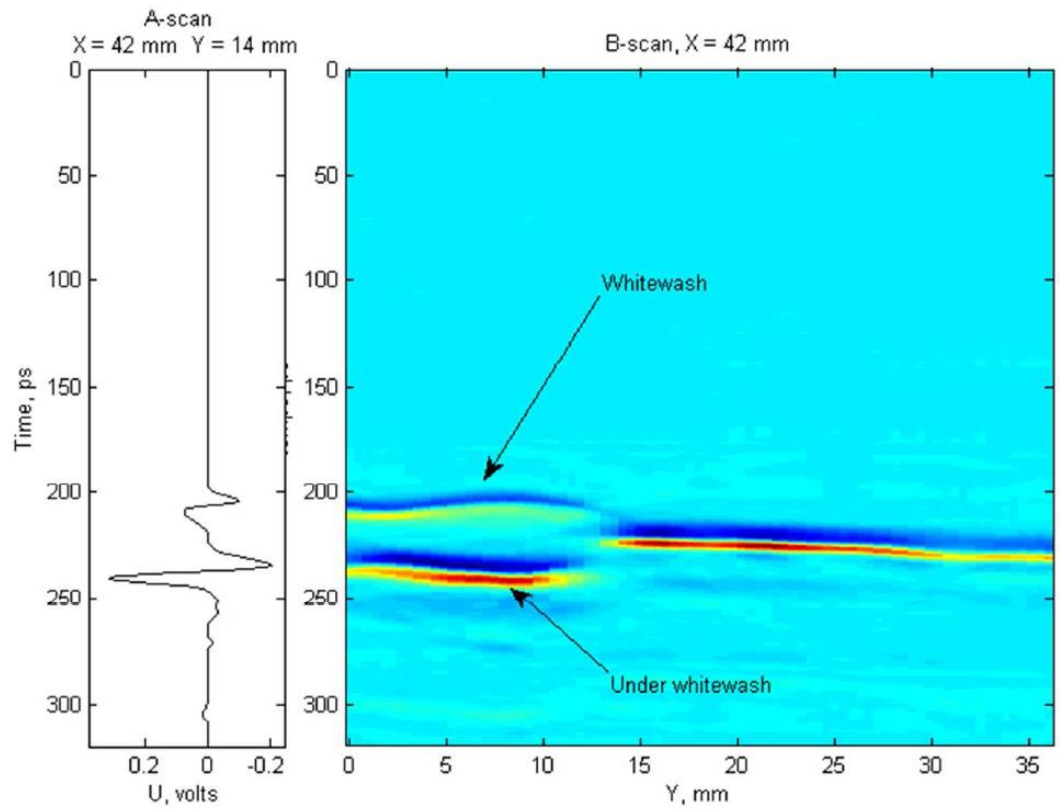

Figure 7: A-scan and B-scan along the dashed line in Figure 6. A-scan of the point $X=42 \mathrm{~mm}, \mathrm{Y}=14 \mathrm{~mm}$ (left). The surface of the whitewash and the hidden layer are indicated in the B-scan (right).

Figure 7 also serves as an example of a limitation of THz-TDI that occurs when an interface is covered by two materials with very different refractive indices. Figure 7 is divided into two parts: on the lefthand side is an A-scan and on the right-hand side, a B-scan. The A-scan was obtained for the coordinate $X=42 \mathrm{~mm}$ and $Y=14 \mathrm{~mm}$. The B-scan was performed along the dashed-line drawn in Figure $6 . \mathrm{In}$ the B-scan, the same interface, with and without the whitewash appears at different depths. This error emphasizes the fact that $\mathrm{THz}$ tomography, as with all the other imaging techniques, requires prior knowledge of the studied object.

In addition to the 2D imaging method described above, 3D imaging can be applied to provide information on the roughness, flatness and layer thickness of the sample structure. This type of 3D imaging is done by specifying a time window on the TD waveform and plotting the time delay corresponding to either the maximum or minimum value within this window as a surface plot.

This technique is Illustrated in application to a Byzantine icon (14 ${ }^{\text {th }}$ century), the so-called Lucas icon, in Freising (Germany). The work was carried out during an archaeometrical campaign (IPERION CH project funded by the European Commission, H2020-INFRAIA-2014-2015, under Grant No. 654028). The main goal of this multi-instrumental campaign was to enhance knowledge of the hidden first 


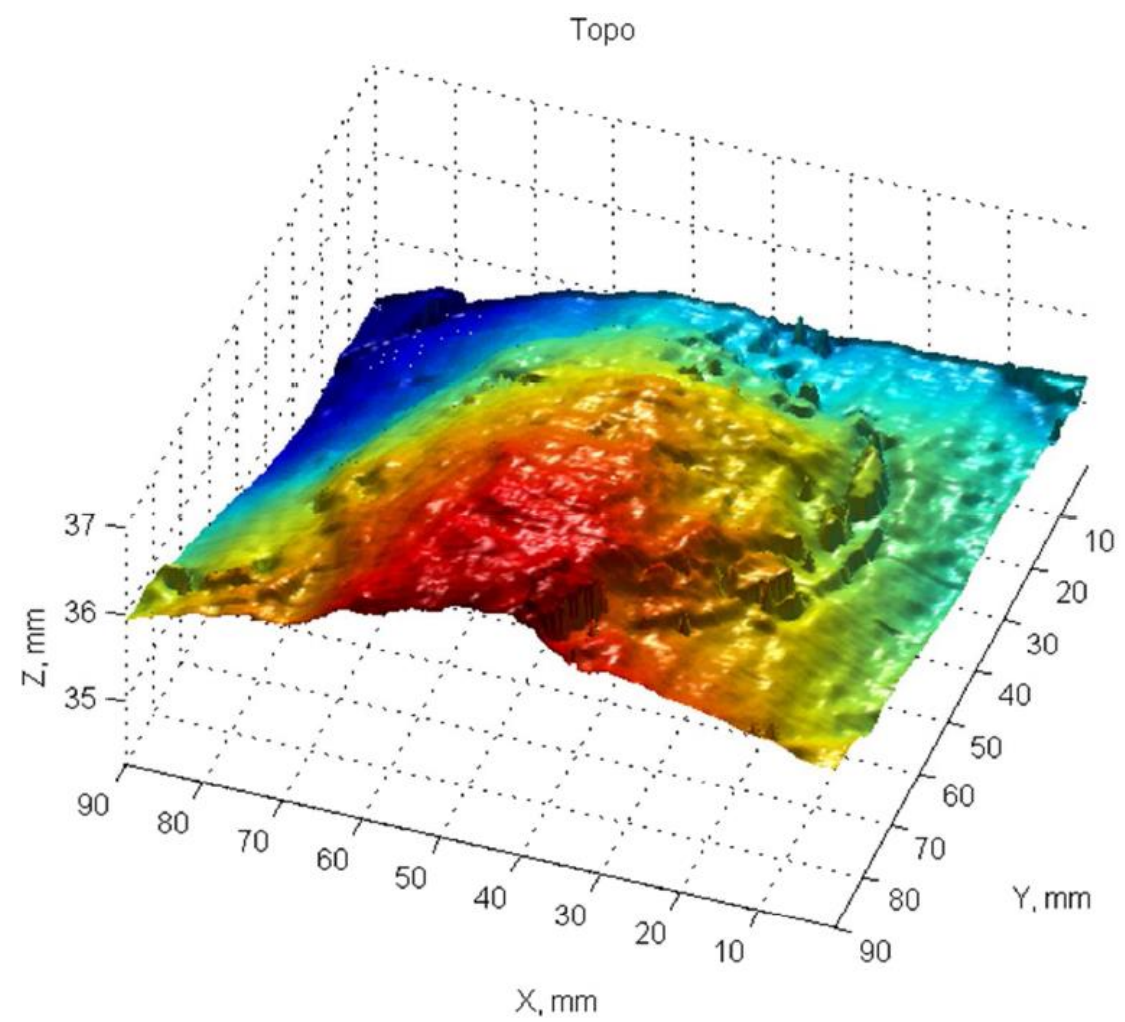

Figure 8: (a) THz topography of the icon surface. The ratio between the three axes is not equal to enhance the topography.

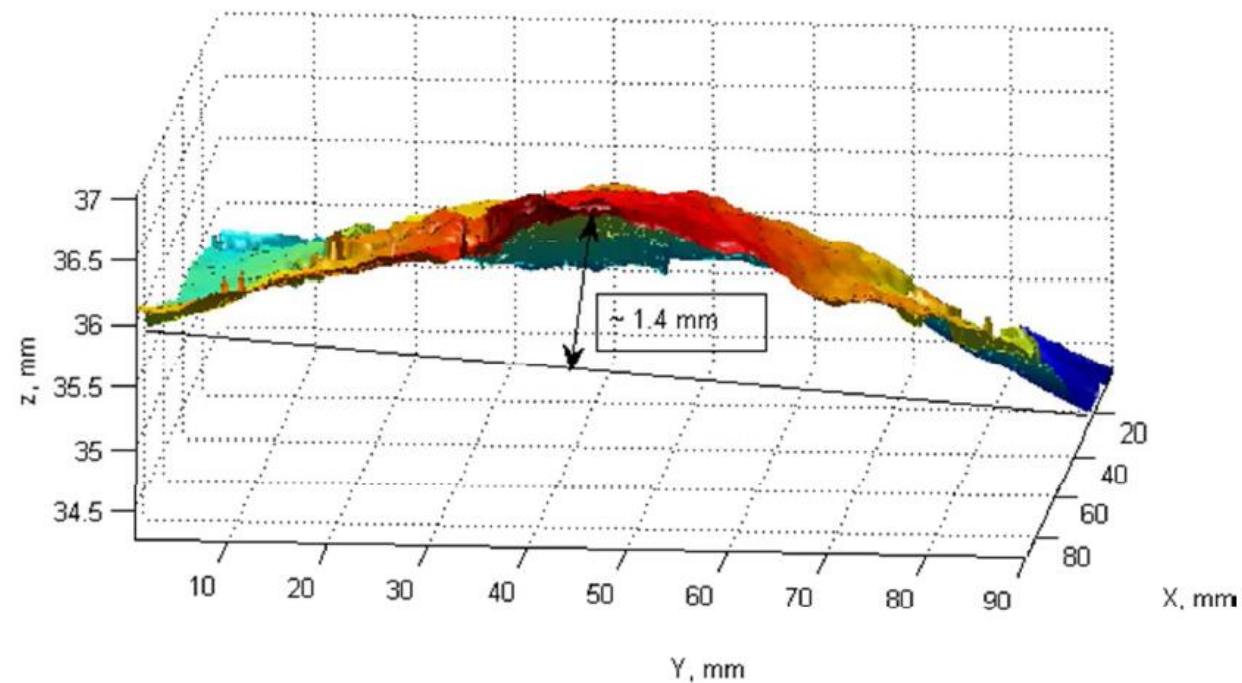

Figure 8: (b) THz topography of the icon surface (side view). The ratio between the three axes is not equal to enhance the topography.

version of the icon and to enable a virtual reconstruction of it. For THz-TDI, the objective was to retrieve a cross section of the icon using nondestructive techniques. The capability for THz-TDI to create 3D images was utilised in this work. The topography of the surface is reconstructed by the use of time of flight in Figure 8. The face of the Virgin (including the halo) is seen to be raised above the 
surrounding area (Figure 8 top). Plus, the artwork has a "natural" curvature, bigger in the center of it (as can be seen in Figure 8 bottom, where the ratio between axes is different to accentuate the curvature of the artwork). The distance between the highest position and the baseline is about 1.4 $\mathrm{mm}$, as given in Figure 8.

\section{Results}

With the prospect of a large restoration campaign on the medieval Doom wall painting in St Thomas' Church (Salisbury, England), a scientific study was carried out to more accurately identify the underlying composition. This wall painting represents the Last Judgment and is located on the arch between the nave and the choir. Documentary evidence reveals that modern painting (19th century) covers much earlier painting (probably 14th century). This ancient layer was covered by several layers of whitewash before being uncovered and retouched/repainted by restorers Clayton and Bell at the end of the 19th century. The original layer is visible to the naked eye under the lacuna (Figure 9).

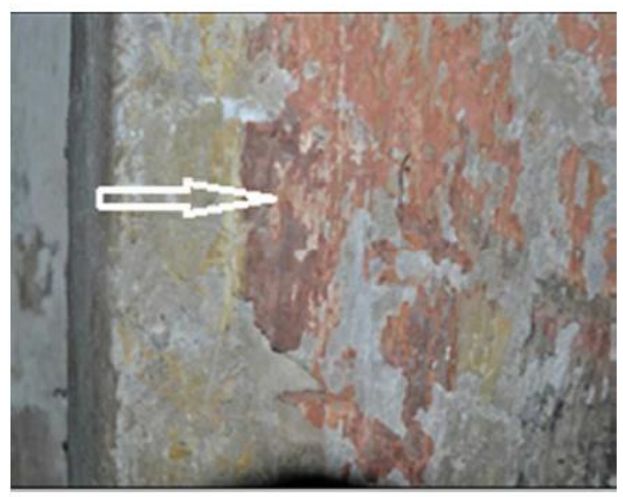

Figure 9: Details of the paint with visible evidence of the original layer (white arrow).

Figure $10(a)$ is a photograph of part of the halo of Jesus, showing the presence of gold paint on the surface. Figure 10 (b) superimposes a C-Scan of the maximum peak-to-peak THz amplitude on top of a greyscale representation of the visible image in Figure 10 (a). A strong reflection appears on the Cscan where there is no visible layer at the surface. On the assumption that there is an intervening covering layer of whitewash (with a refractive index of 1.6 [20]), the depth of the underlying layer giving rise to the strong reflection can be estimated to be around $100 \mu \mathrm{m}$.

A physical sample was taken from the region of interest, a cross section of which is presented in Figure 11. From this, it appears that a highly reflective layer of gold leaf exists under the surface. The surface layer is made of a thick black layer covered by gold paint. A thin lime whitewash layer covers the layer of gold leaf, which lies on a red preparation layer composed of minium and red ochre over a thin pink layer. The last layer is made of lime whitewash. From this sample, the gold leaf is at a depth close to $100 \mu \mathrm{m}$, which fits perfectly with the estimation obtained by the $\mathrm{THz}$ technique.

Under the framework of the European Project Iperion CH (H2020-INFRAIA-2014-2015, under Grant No. 654028) a campaign was undertaken with different experimental techniques: $\mathrm{THz}$ imaging, Stimulated Infrared Thermography (SIRT) and Digital Holographic Speckle Pattern Interferometry (DHSPI). The measurements were carried out in the 12th century Church of St. Gregory the Great, in Morville, Shropshire, UK. Documentary evidence suggests the existence of a painting on the south wall of the church, which has been covered with plaster and/or paint layers. However, the description is ambiguous with regard to the painting's exact location on the south wall. 


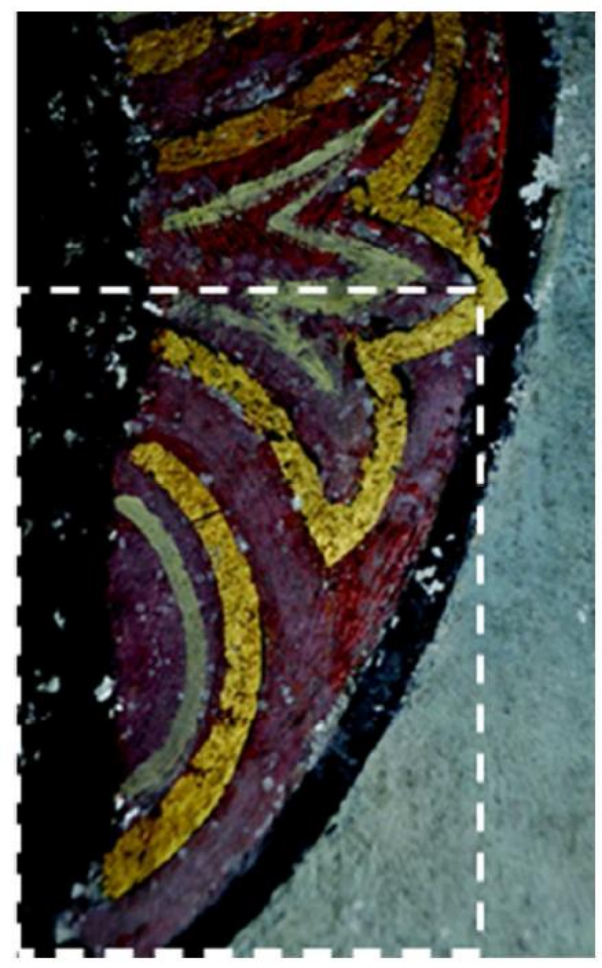

Figure 10: (a) Part of the halo of Jesus

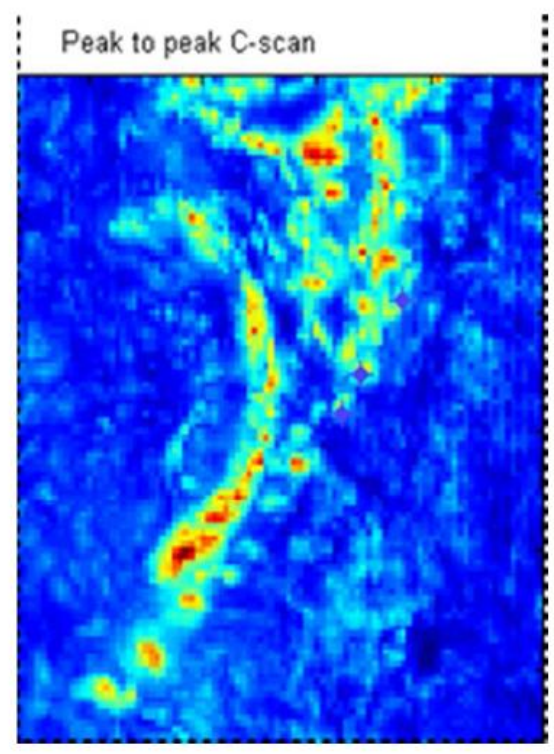

Figure 10: (b) Overlap of the visible photography and the peak to peak C-scan of the ROI. The area in red is symptomatic of a strong reflection. 


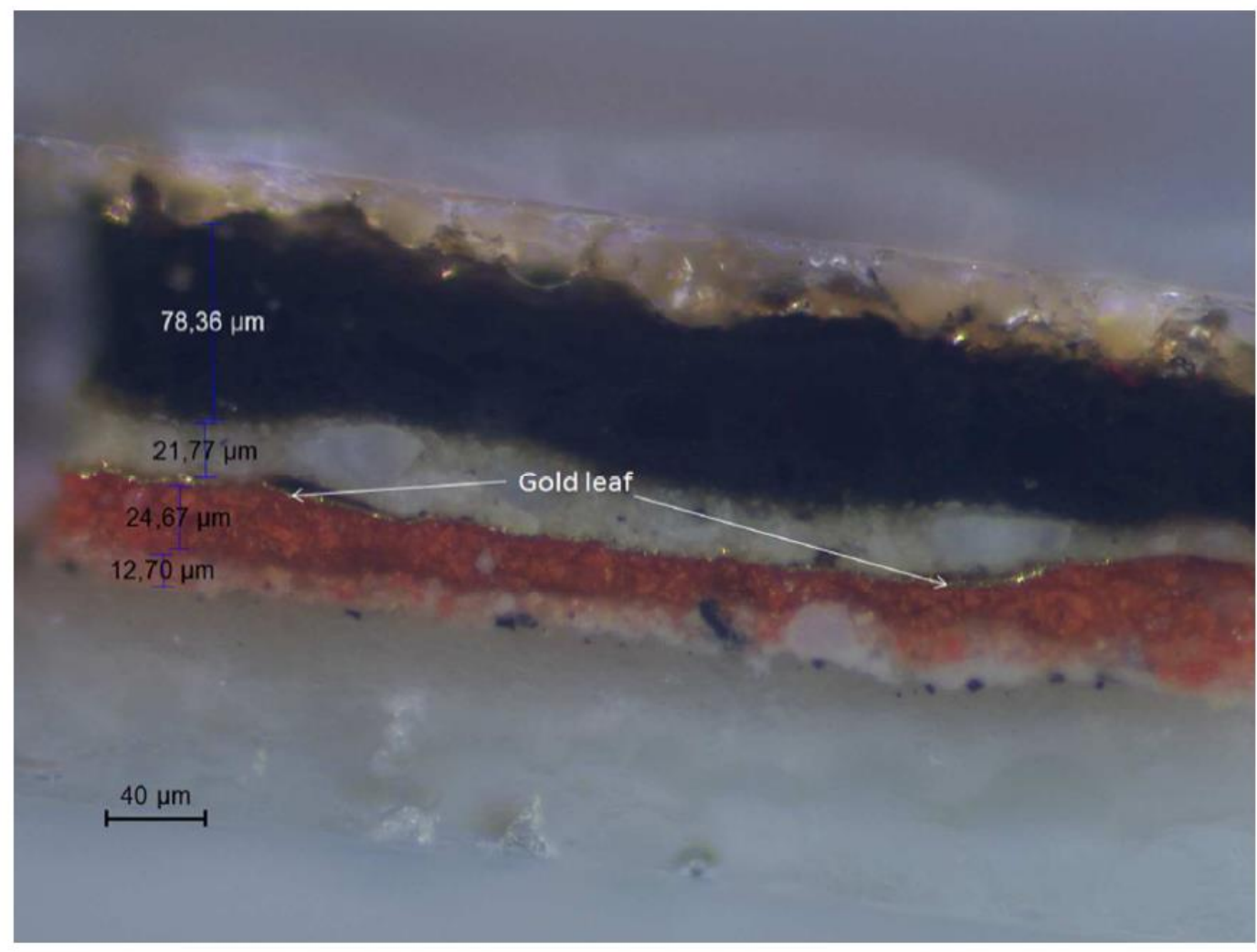

Figure 11: Cross section of a sample taken on the halo of Jesus.

Stimulated infrared thermography was performed using a system developed by LRMH and the University of Reims Champaign Ardennes. DHSPI was carried out using a system developed by IESL/FORTH.

Results delivered by the three techniques are consistent and were able to distinguish areas with structural defects and deterioration from those that were more solid ([33]). While thermography and DHSPI cannot provide direct images of concealed paintings, the results show that these techniques can provide valuable clues to their location that can be used to target subsequent detailed but more time-consuming terahertz imaging. Additionally, they can be used to identify cracks, voids and delaminations that can give rise to artifacts in terahertz images, giving scope for their removal in subsequent processing. As an example, Figure 12 shows SIRT and DHSPI images from a $690 \mathrm{~mm} \times 510$ $\mathrm{mm}$ area of the south wall of the church. The bright red and yellow regions in the SIRT image and the structured pattern and large number of localized anomalies in the DHSPI image are strongly suggestive of hidden structure within the wall, potentially due to the presence of sub-surface paint layers. This conclusion is borne out by the $\mathrm{THz}$ B-scan from the same area of wall shown in Figure 13, which indicates the presence of three internal layers, consistent with localized areas of pigment within the wall painting.

A final example of results obtained with the $\mathrm{THz}$ system comes from experiments carried out in a Thermal-Hydraulic-Chemical-Mechanical (THCM) study to improve understanding of the behavior of plasterboard warmed on one face to simulate normalized fire (ISO 834 [34]). Panels of gypsum ( $\beta$ calcium sulfate, water to plaster mass ratio (W/P) equal to $0.65,40 \mathrm{~mm}$ thick) are taken as samples due to their widespread use as a passive fire protection. The relative fragility of gypsum boards is a benefit because the scenarios of damage are rich enough for the data to be transposable to more complex configurations. During equivalent fire, mechanical damage of the structure by the emergence 
of a network of cracks is enhanced by five distinctive and polymorphous phase changes. Four of these phase changes are endothermic and the other one is exothermic, with or without mass transfer [3536].

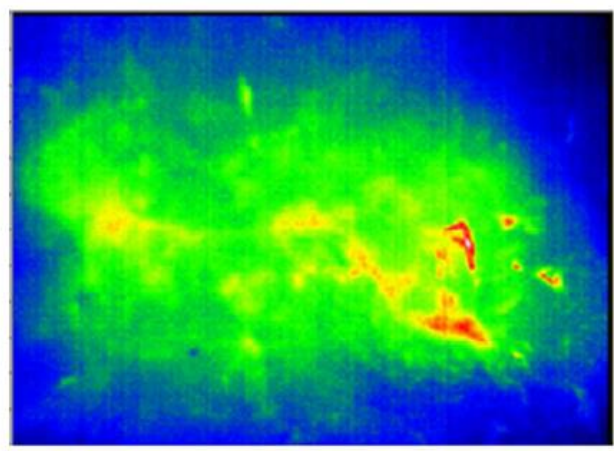

(a)

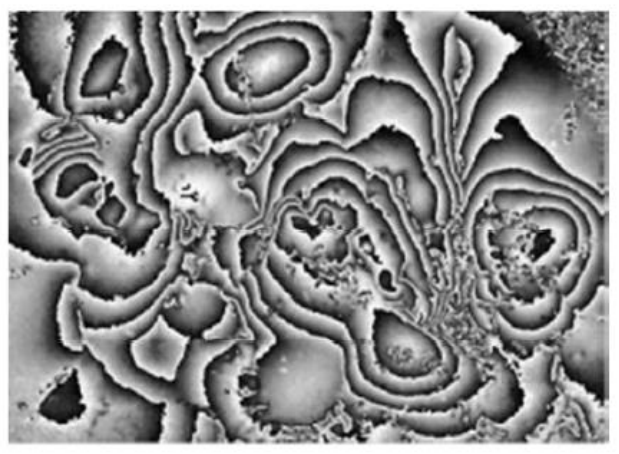

(b)

Figure 12: (a) SIRT image and (b) DHSPI image from the south wall of the Church of St. Gregory the Great, Morville, Shropshire, UK.

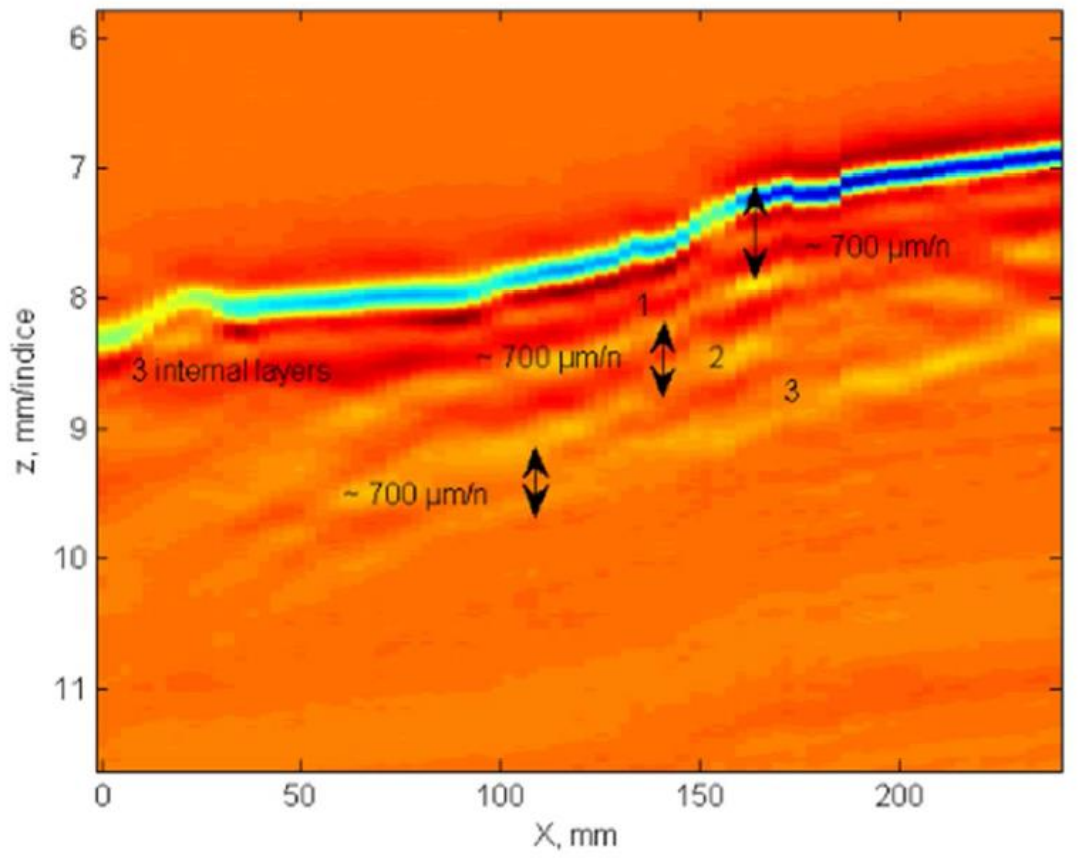

Figure 13: THz B-scan from section C - note the presence of 3 internal layers, consistent with localized areas of pigment within the wall painting.

Thermal analyses reveal the presence of three out of the four well-known phenomena that occur when gypsum is heated (equations 1-4) [35-38]). First, an endothermic dehydration in two steps occurs between 80 and $200^{\circ} \mathrm{C}$ (Equations 1 and 2). Then, an anhydrite III to anhydrite II exothermic phase transition is observed between 350 and $400^{\circ} \mathrm{C}$ (Equation 3). The last reaction usually takes place at temperatures above $1180^{\circ} \mathrm{C}$ (Equation 4) and was not observed in these experiments. The endothermic phenomenon observed between 600 and $750^{\circ} \mathrm{C}$ is due to decarbonation of $\mathrm{CaCO}_{3}$ impurities (Equation 5) [37-38]. 
$\mathrm{CaSO}_{4}, 2 \mathrm{H}_{2} \mathrm{O} \rightarrow \mathrm{CaSO}_{4}, 0.5 \mathrm{H}_{2} \mathrm{O}+1.5 \mathrm{H}_{2} \mathrm{O}$

$\mathrm{CaSO}_{4}, 1.5 \mathrm{H}_{2} \mathrm{O} \rightarrow \mathrm{CaSO}_{4}(\mathrm{III})+0.5 \mathrm{H}_{2} \mathrm{O}$

$\mathrm{CaSO}_{4}(\mathrm{III}) \rightarrow \mathrm{CaSO}_{4}(\mathrm{II})$

$\mathrm{CaSO}_{4}(\mathrm{II}) \rightarrow \mathrm{CaSO}_{4}(\mathrm{I})$

$\mathrm{CaCO}_{3} \rightarrow \mathrm{CaO}+\mathrm{CO}_{2}$

The internal structure of such material is well known by destructive techniques. Illustrations of the local chemical compound are given through the thickness of the board versus time in Figure 14. The challenge here is to achieve the same results through non-destructive examination.

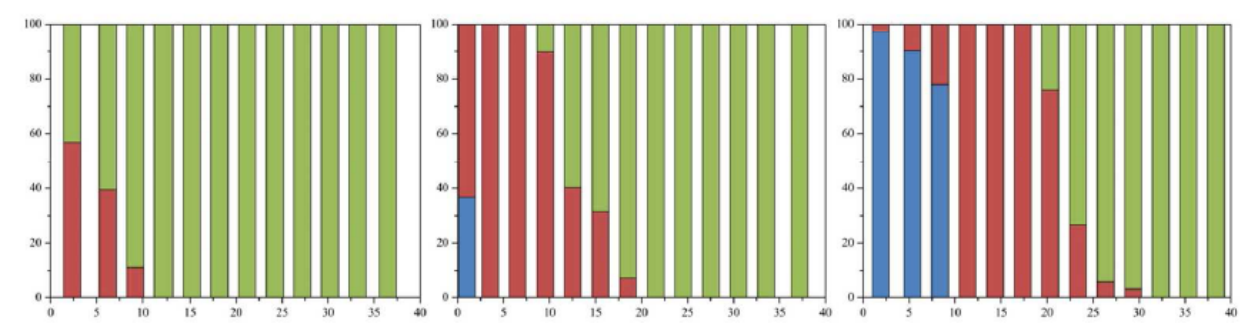

After 75 min testing

After 105 min testing

After 150 min testing

Figure 14: Identification of the local chemical composition from exposed side ( $40 \mathrm{~mm})$ to non-exposed side $(0 \mathrm{~mm})$ versus time of a gypsum board $(W / P=0.65)$ heated on one face under ISO 834 normalized fire. Results are deducted by mean of a destructive technique. Blue color represents gypsum chemical phase, red color represents semi-hydrate chemical phase and green color represents anhydrite chemical phase,

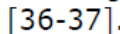

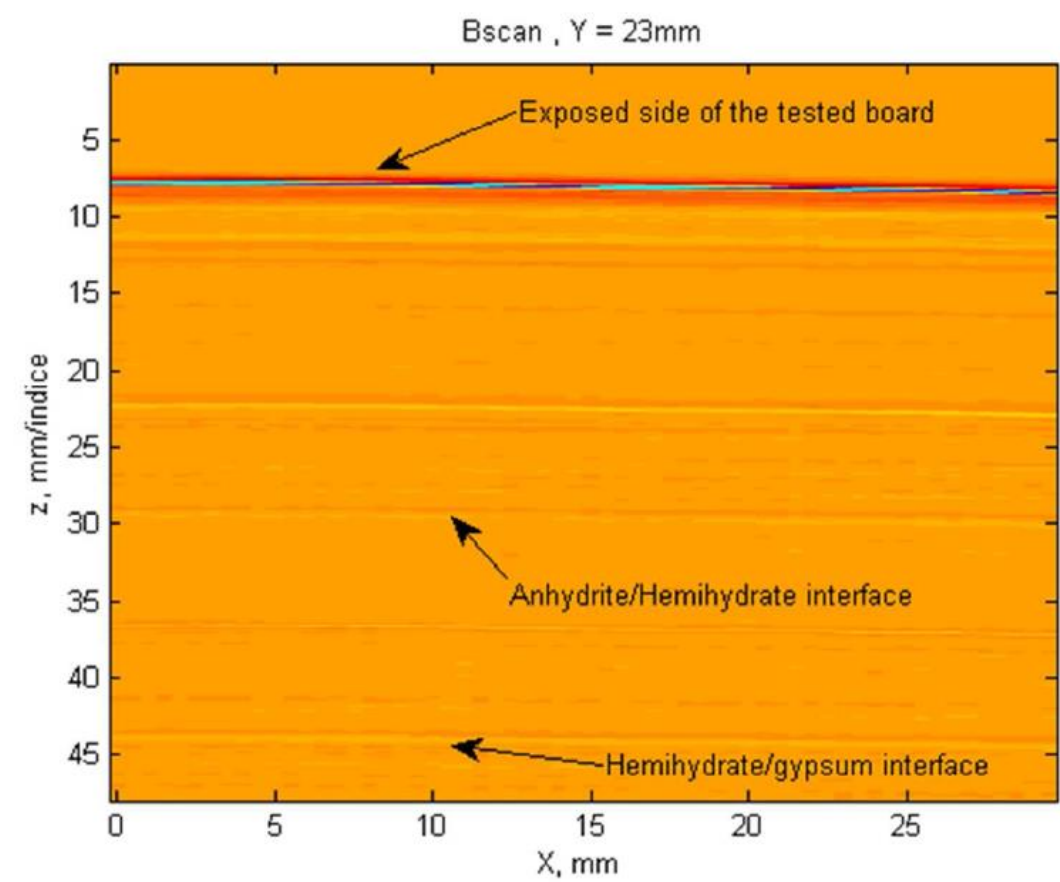

Figure 15: THz B-scan from gypsum panel after 2 hours testing under normalized fire. Note the presence of 2 internal layers is consistent with localized layers of phase changes with a refractive index of 1.6 ([35-381). 
During heating, it is not possible to scan the surface with the $\mathrm{THz}$ system without damaging the equipment. The protocol is to stop the experiment after a fixed time and to scan the board when it is cold.

Figure 15 shows a B-scan of a board after 2 hours testing. The exposed surface is on the top. The surface of the sample appears as a red-blue line at $7 \mathrm{~mm}$ (the vertical axis represents the optical distance; the refractive index is taken as 1 in air and between 1.6 and 1.8 in plaster [9]). Two layers are visible (black arrows on Figure 15) at a depth corresponding to water content and phase changes of gypsum for this heating time ([35-36]). Such results are in good agreement with destructive testing techniques.

\section{Conclusion}

This study demonstrates the capabilities of THz-TDI for characterizing immovable cultural heritage materials, understanding decay processes and improving conservation of cultural objects. Specifically for conservation science, the capacity of $\mathrm{THz}$ to image the internal structure of such materials with non-ionizing radiation is of great benefit, compared to X-rays, which require skilled personnel and special facilities such as radiation-shielded rooms. Moreover, THz-TDI is contact-free and gives information on a wide variety of optically opaque and non-conducting materials.

As THz-TDI can be carried out in reflection, there is no need to access both sides of an object. THz imaging can provide information at depth and $\mathrm{THz}$ data contains spectral information that can potentially be used to identify different materials.

The versatility of THz-TDI is one of the key points for its success in important applications in conservation science.

The combination of THz-TDI with other nondestructive techniques, such as SIRT and DHSPI, is very promising, with the capability to provide information simultaneously on the structure of materials and the mechanical activity of identified layers.

\section{Acknowledgments}

The authors would like to thank V. Tornari and M. Andriankis from IESL/FORTH for conducting the DHSPI measurements, V. Detalle and E. Bourguignon for the SIRT measurements, and to gratefully acknowledge the financial support of the Fondation des sciences du patrimoine/LabEx PATRIMA ANR10-LABX-0094-01, the European projects Charisma (grant no 228330) and IPERION CH (grant $n^{\circ}$ : 654028), and the University of Cergy-Pontoise.

\section{Bibliography}

[1] K. E. Peiponen, J. A. Zeitler and M. Kuwata-Gonokami, Terahertz Spectroscopy and Imaging, Vol, 171, Springer Series in Optical Science, Springer Heidelberg New York Dordrecht London, 641 pages, 2013.

[2] L. Chusseau, J. Demaison, J.L. Coutaz, Optoélectronique térahertz, EDP Sciences. 240 pages, 2008.

[3] J. Labaune, E. Abraham, Le rayonnement térahertz pour l'analyse des matériaux du patrimoine culturel, Technique de l'ingénieur, RE 213, 28 pages, 2013. 
[4] E. Pickwell-MacPherson, V. Wallace, Terahertz pulsed imaging - a potential medical imaging modality? Photodiagnosis and Photodynamic Therapy. 6-2, 128-134, 2009.

[5] A. G. Davies, A. D. Burnett, W. Fan, E. H. Linfield, et J. E. Cunningham et al, Terahertz spectroscopy of explosives and drugs. Materials Today.11-3, 18-26, 2008.

[6] W. Köhler, U. Klotzbach, E. Beyer, S. Winnerl, M. Helm, F. Rutz, C. Jördens, M. Koch, H.Leitner, «Non-Destructive Investigation of Paintings with THz-Radiation», Proc. Europ. Conf. Non Destructive Testing, 1-7, 2006.

[7] K. Fukunaga, I. Hosako, Thz Spectroscopy For Analysis Of Paintings 2007. Annual Report Conference on Electrical Insulation and Dielectric Phenomena, 715-718, 2007.

[8] K. Fukunaga, Y. Ogawa, S. Hayashi, Application of terahertz spectroscopy for character recognition in a medieval manuscript. IEICE Electron. Express, 5, 223-228, 2008.

[9] G. C. Walker, J. W. Bowen, J. B. Jackson, J. Labaune, G. Mourou, M. Menu, W. Matthews, Subsurface terahertz imaging through uneven surfaces: visualizing Neolithic wall paintings in Çatalhöyük. Optics express, 21- 7, 8126-8134, 2013.

[10] J. B. Jackson, M. Mourou, J.F. Whitaker, I.N. Duling III, S.L. Williamson, M. Menu, G.A. Mourou, Terahertz imaging for non-destructive evaluation of mural paintings. Optics Communications, 281-4, 527-532, 2008.

[11] K. Fukunaga, M. Picollo, THz techniques as a complementary non-invasive diagnostic method for the study of medieval panel paintings. IEEE International Conference on Solid Dielectrics, Bologna, Italy, 493-496, 2013.

[12] E. Abraham, A. Younus, A. El Fatimy, J. C. Delagnes, E. Nguéma, et P. Mounaix, Broadband terahertz imaging of documents written with lead pencils. Optics Communications, 282-15, 31043107, 2009.

[13] A.J.L Adam, P.C.M. Planken, S. Meloni, J. Dik, Terahertz imaging of hidden paint layers on canvas. Optics Express, 17, 3407-3416, 2009.

[14] F. Cesarani, M. C. Martina, A.Ferraris, R. Grilletto, R. Boano, E. Fiore Marochetti, A. M. Donadoni, G. Gandini, Whole-body three-dimensional multidetector CT of 13 Egyptian human mummies. American Journal of Roentgenology, 180-3, 597-606, 2003.

[15] H. Hoffman, W.E. Torres, R.D. Ernst, Paleoradiology: Advanced CT in the evaluation of nine Egyptian mummies. Radiographics, 22, 377-385, 2002.

[16] A. Cosentino, C.L. Koch, Dandolo, A. Cristaudo, P. Uhd Jepsen, Diagnostics pre and post Conservation on a 14th Century Gilded Icon from Taormina, Sicily. Available online: http://www.econservation.org/issue-3/49-Diagnostics-on-a-14th-Century-Gilded-Icon-from-Taormina

[17] M. Picollo, K. Fukunaga, J. Labaune, Obtaining noninvasive stratigraphic details of panel paintings using terahertz time domain spectroscopy imaging system. Journal of Cultural Heritage, 16, 73-80, 2015.

[18] J. Dong, J. B. Jackson, M. Melis, D. Giovannacci, G.C. Walker, A. Locquet, J.W. Bowen, D.S. Citrin, D.S., Terahertz frequency-wavelet domain deconvolution for stratigraphic and subsurface investigation of art painting. Optics Express 24, 26972-26985, 2016. 
[19] K. Krügener, M. Schwerdtfeger, S.F. Busch, A. Soltani, E. Castro-Camus, M. Koch, W. Viöl, Terahertz meets sculptural and architectural art: Evaluation and conservation of stone objects with Tray technology. Science Report, 5, 14842, 1-12, 2015.

[20] G.C Walker, J. B. Jackson, D. Giovannacci, J. W. Bowen, B. Delandes, J. Labaune, G. Mourou, M. Menu, V. Detalle, Terahertz analysis of stratified wall plaster at buildings of cultural importance across Europe. Proceedings of the SPIE 8790, Optics for Arts, Architecture, and Archaeology IV, Munich, Germany; Volume 8790, 1-8, 2013.

[21] D. Giovannacci ; D. Martos-Levif ; G. C. Walker ; M. Menu and V. Detalle, Terahertz applications in cultural heritage: case studies. Proceedings of the SPIE 9065, Fundamentals of Laser Assisted Microand Nanotechnologies 2013 (FLAMN 2013), St Petersbourg, Russia, Volume 9065, 906510-1-9065108, 2013.

[22] T. Bardon, R.K. May, P.F. Taday, M. Strlic, Material characterization of historical parchment using terahertz time-domain spectroscopy. Proceedings of 39th International Conference on Infrared, Millimeter, and Terahertz waves (IRMMW-THz), Tucson, AZ, USA, 1-2, 2014.

[23] T. Bardon, R.K. May, P.F. Taday, M. Strlic. Systematic study of terahertz time-domain spectra of historically informed black inks. Analyst, 138, 4859-4869, 2013.

[24] T. Kurabayashi, S. Sakai, K. Fujino, Sub-terahertz imaging of painted steel. Proceedings of the 35th International Conference on Infrared, Millimeter and Terahertz (IRMMW-THz), Rome, Italy, p. 1-2, 2010.

[25] R.F. Anastasi, E.I. Madaras, Terahertz NDE for under paint corrosion detection and evaluation. Proceedings of AIP, 820, 515-522, 2006.

[26] H. Zhao, Hui Zhao, D. Wu, H. Zhan, Q. Sun K. Zhao. Detection of iron corrosion by terahertz time domain spectroscopy. Proceedings of the SPIE 9795, Selected Papers of the Photoelectronic Technology Committee Conferences, Hefei, Suzhou, and Harbin, China, p. 97953, 2015.

[27] J. Labaune, J.B. Jackson, S. Pagès-Camagna, I.N. Duling, M. Menu, G.A. Mourou, Papyrus imaging with terahertz time domain spectroscopy. Applied Physics. A, 100, 607-612, 2010.

[28] G. C. Walker, J. Labaune, J. W. Bowen, J.B. Jackson; S. Hadjiloucas, G. Mourou, Deconvolution: Imaging the unturned page. Proceedings of the 2011 36th International Conference on Infrared, Millimeter and Terahertz Waves (IRMMW-THz), Houston, TX, USA, 1-2, 2011.

[29] M. Koch S. Hunsche, P. Schuacher, M. C. Nuss, J. Feldmann, J. Fromm . THz-imaging: A new method for density mapping of wood. Wood Science Technology, 32, 421-427, 1998.

[30] R.M Groves, B. Pradarutti, E. Kouloumpi, W. Osten, G. Notni, 2D and 3D non-destructive evaluation of a wooden panel painting using shearography and terahertz imaging. NDT\&E International, 42, 543-549, 2009

[31] J.B. Jackson, M. Mourou, J. Labaune, J.F. Whitaker, I.N. Duling, S.L. Williamson, C. Lavier, M.Menu, G.A. Mourou, G.A.Terahertz, pulse imaging for tree-ring analysis: A preliminary study for dendrochronology applications. Measurement Science and Technology, 20-7, 075502, 1-10, 2009.

[32] G.C. Walker, J.W. Bowen, J. Labaune, J.-B. Jackson, S. Hadjiloucas, J. Roberts, G. Mourou, and M. Menu, “Terahertz deconvolution”, Optics Express, 20 (25), 27230-27241, 2012. 
[33] J.W. Bowen, D. Giovannacci, V. Tornari, E. Bourguignon, D. Brissaud, M. Andrianakis, V. Detalle, An investigation into the complementarity of $\mathrm{THz}$ imaging, stimulated infrared thermography and DHSPI for the imaging of concealed wall paintings, OTST 2017, 2-7 April 2017, University College London, London, UK, 2017.

[34] ISO 834-1, Fire Resistance Tests - Elements of Building Construction - Part 1: General requirements, 1999.

[35] D Giovannacci, V. Tornari, M. Andrianakis, Y. Mélinge, D. Brissaud, J. Wassermann, L. Cristofol Mise au point d'une plateforme expérimentale CND de caractérisation de la structure interne de plâtre soumis au feu. Proceedings of RUGC 35ème Rencontres universitaires de génie civil, Nantes, France, 249-252, 2017.

[36] A. Rojo, Y. Mélinge, O. Guillou. Kinetics of internal structure evolution in gypsum board exposed to standard fire, Journal of Fire Sciences, 31-5, 395-409, 2013.

[37] Y. Mélinge, K.S. Nguyen, C. Daiguebonne, O. Guillou, S. Freslon, C. Lanos. One-dimensional time study of the dehydration of plasterboards under standard fire condition (ISO 834): Thermo-Chemical Analysis, Journal of Fire Sciences, 29-4, 299 - 316, 2011.

[38] A. Rojo. Etude de la structuration et du comportement du gypse sous condition incendie. PhD thesis, INSA de Rennes, 2013. 